\title{
An Arbitrary Polynomial Chaos-Based Approach to Analyzing the Impacts of Design Parameters on Evacuation Time under Uncertainty
}

\author{
QIMIAO XIE ${ }^{1}$, SHOUXIANG LU ${ }^{1}$, DANIEL CÓSTOLA ${ }^{2}$, and JAN L.M. HENSEN ${ }^{2}$ \\ ${ }^{1}$ State Key Laboratory of Fire Science \\ University of Science and Technology of China \\ Hefei, Anhui 230027, China \\ ${ }^{2}$ Building Physics and Services \\ Eindhoven University of Technology \\ Eindhoven $5600 \mathrm{MB}$, Netherlands
}

\begin{abstract}
In performance-based design of buildings, much attention is paid to design parameters by fire engineers or experts. However, due to the time-consuming evacuation models, it is computationally prohibitive to adopt the conventional Monte Carlo simulation (MCS) to examine the effects of design parameters on evacuation time under uncertainty. To determine suitable design parameters under uncertainty with the reduced significantly computational cost, an arbitrary polynomial chaos-based method is presented in this paper. Arbitrary polynomial chaos expansion is used to construct surrogate models of evacuation time based on complex evacuation models. Afterwards, simple analytical method can be adapted to calculate the mean, standard deviation of evacuation time and Sobol sensitivity indices based on the arbitrary polynomial chaos coefficients. Moreover, the distribution of evacuation time can be generated by combining Latin hypercube sampling (LHS) with the obtained surrogate model. To demonstrate the proposed method, a hypothetical single-storey fire compartment with two exits is presented as a case in accordance with the Chinese code GB50016-2012, evaluating the impact of exit width on evacuation time under uncertain occupant density and child-occupant load ratio. And results show that the proposed method can achieve the distribution of evacuation time close to that from the MCS while dramatically reducing the number of evacuation simulations. When exit width per 100 persons is designed between $0.1 \mathrm{~m}$ and $0.5 \mathrm{~m}$, the uncertainty of evacuation time is severely affected by exit width, which is more significant in smaller exit width. However, exit width has a small effect on Sobol sensitivity indices, the reliability level of a certain safety factor, and safety factor at a certain reliability level.
\end{abstract}

KEYWORDS: performance-based design, egress, evacuation time, uncertainty analysis, risk assessment, statistics

\section{INTRODUCTION}

Occupant evacuation is extremely complex, which is influenced by the physiological, psychological, and sociological aspects of evacuees as well as external environment [1]. Due to the randomness of fire occurrence and the variability of occupants' characteristics, there exist many uncertain factors, such as premovement time, occupant density, occupant type, familiarity with exits, and occupant mobility etc. Thus evacuation time is highly uncertain for a certain specific building. Traditionally, prescriptive fire protection design codes adopt safety factors to deal with uncertain factors related to crowd evacuation [2]. However, with the help of prescriptive building fire protection codes, there is still a lack of specific guidance about the selection of safety factors. Meanwhile, it is also difficult to determine the safety performance of buildings and optimal design parameters using prescriptive building codes. In order to address the problems above, it is necessary to employ probabilistic methods to examine the effect of design parameters on evacuation time under uncertainty.

During the last few decades, research has been widely conducted about the effect of design parameters on evacuation time. Based on the Monte Carlo simulation (MCS) of the STEPS and EXIT89 evacuation models, Meacham et al. [3] suggested that significant uncertain parameters for evacuation time may be associated with the geometry of buildings. Fang et al. [4] employed a multi-grid evacuation model to investigate the effect of door width on evacuation time and assumed that door width has a more significant influence on evacuation time in fire situations than normal conditions. Zhao et al. [5] adopted a cellular 
automaton evacuation model to conduct the performance-based design of building exits in deterministic scenarios and suggested that the layout of exits should be symmetrical and the distance between exits should be designed using optimization techniques. In order to improve building designs for a certain deterministic scenario with the reduced computational cost, Tavares et al. [6] combined the Building EXODUS evacuation model and design of experiments to obtain the deterministic response surface of evacuation time. Afterwards, numerical optimization methods are applied to determine the optimal design parameter. In current building regulations, exit location is usually determined by the maximum travel distance in a certain specified scenario. In order to find an alternative method to determine suitable exit location, Tavares et al. [7] compared evacuation times from the exit location determined by the relative distance between exits and the maximum travel distance through a case study, and the result is that evacuation time from exit location determined by the relative distance between exits is smaller, which indicates exit location can also be determined by the relative distance between exits. It can be seen that Ref. [6] and [7] focus on the optimization of design parameters with deterministic values of uncertain parameters using the deterministic response surface method and the comparison of deterministic evacuation scenarios, and thus the determination of design parameters under uncertainty are paid attention to in this paper. Furthermore, in order to compute the reliability of evacuating successfully with the low computational cost, Cornelius et al. [8] adopted the adaptive deterministic response surface method to build the surrogate models of fire and evacuation models.

As the discussion above, the impacts of design parameters on evacuation time are mainly studied in the case of assigning deterministic values to uncertain parameters such as occupant density. However, evacuation time is highly uncertain due to the randomness of fire occurrence and human characteristics. Meanwhile, due to the complexity of evacuation models, it is computationally expensive to adopt the conventional MCS to investigate the effects of design parameters on evacuation time under uncertainty. Stochastic response surface methods can be used to deal with the output uncertainty caused by the uncertainty in input parameters with the low computational cost [9]. And the arbitrary polynomial chaos expansion proposed by Witteveen et al. [10] is one of the stochastic response surface methods, which can handle arbitrary distributions of input parameters only with limited statistical moments. Thus, in order to take the reduced significantly computational cost to analyze the effects of design parameters on evacuation time under arbitrary uncertainty, the arbitrary polynomial chaos-based method is proposed here. Based on results of computationally expensive evacuation models, arbitrary polynomial chaos expansion can be used to construct surrogate models of evacuation time with uncertain input parameters. And then, the mean and standard deviation of evacuation time as well as Sobol sensitivity indices can be calculated analytically based on the arbitrary polynomial chaos coefficients. Furthermore, Latin Hypercube sampling (LHS) can be applied to uncertain parameters to generate input samples. Afterwards, the surrogate model of evacuation time is performed on these input sample points to achieve the distribution of evacuation time.

\section{ARBITRARY POLYNOMIAL CHAOS-BASED APPROACH}

The procedure for the arbitrary polynomial chaos-based approach, which is used to investigate the influence of design parameters on evacuation time under uncertainty with the reduced significantly computational cost, is shown in Fig. 1.

\section{Evacuation Models and Input Parameters}

Evacuation time can be calculated by simple calculations or complex computer evacuation models [11]. Simple calculations assume a number of simplifications in the evacuation process, which are based on observations and evacuation experiments. In order to obtain more accurate results, complex computer evacuation models are developed. According to different classification methods, computer evacuation models can fall into microscopic and macroscopic models; discrete and continuous models; deterministic and stochastic models; rule-based and force-based models; high and low fidelity models [12]. Zheng et al. [13] reviewed seven methodological approaches for crowd evacuation and suggested that different modeling approaches should be combined together to simulate occupant evacuation. Ronchi et al. [14] analyzed the advantages and limitations of computer evacuation models and indicated that computer evacuation models should be based on reasonable modeling assumptions, embedded sub-algorithms and treatment of model uncertainty. FDS+Evac computer model is an agent-based social force model, which is also a stochastic evacuation model. Moreover, FDS+Evac results have already been compared with some 
other evacuation models and simulation results of evacuation time are quite similar [15]. Thus, in this paper, the FDS+Evac model is chosen to predict necessary evacuation time samples, which can be used to construct the surrogate model of evacuation time.

Input parameters of evacuation models can be classified into two types: design parameters and uncertain parameters. For the performance-based fire protection design of buildings, design parameters are generally related to the building geometry, which contain exit width, exit location the number of exits and so on. It is expensive to change these design parameters once buildings are built. Thus, design parameters should be chosen prudently by fire protection engineers or experts. Generally the ranges of design parameters can be assumed according to the building codes, building geometry and occupancy type. Due to a high degree of uncertainty in fire occurrence, it is difficult to determine initial values of some parameters associated with crowd evacuation such as occupant density, occupant type and so on, which are considered as uncertain parameters due to the incomplete knowledge. Generally, the distributions of uncertain parameters can be assumed based on the literature, observations, codes and recommendations by experts etc. [16]. Through the analysis above, it can be seen that evacuation time is determined by design parameters and influenced by uncertain parameters, whose uncertainty should be quantified.

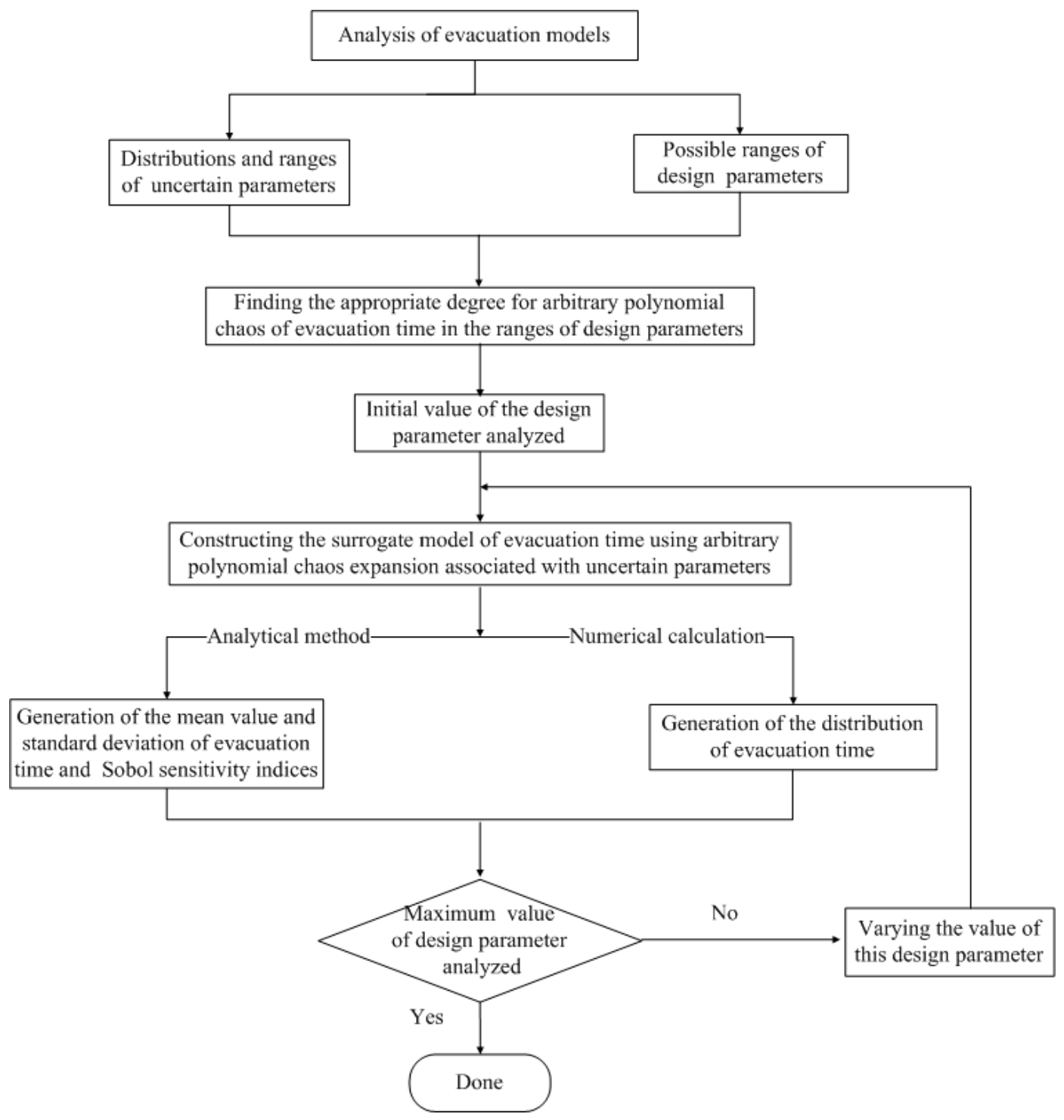

Fig. 1. Procedure for impacts of design parameters on evacuation time under arbitrary uncertainty. 


\section{Arbitrary Polynomial Chaos Expansion}

Arbitrary polynomial chaos is the combination of polynomial chaos expansion and Gram-Schmidt orthogonalization [10], which can be used to address the output uncertainty affected by uncertain parameters with arbitrary distributions, such as truncated standard distributions and histogram distributions etc. In order to quantify the uncertainty of evacuation time, the predictive model of evacuation time associated with independent uncertain input parameters can be expressed as follows.

$$
T_{e}=F(\mathbf{x}) \quad \mathbf{x}=\left\{x_{1}, x_{2}, \cdots, x_{n}\right\}
$$

Where $T_{e}$ is evacuation time; $n$ is the number of uncertain parameters; $\mathbf{x}=\left\{x_{1}, x_{2}, \ldots, x_{n}\right\}$ is the vector set of independent uncertain parameters associated with evacuation time.

According to Wiener [17], the output $T_{e}$ can be expressed in mathematical series with regard to independent input variables $x_{1}, x_{2}, \mathrm{~L}, x_{n}$, as shown in Eq. 2 .

$$
\begin{aligned}
& T_{e}\left(x_{1}, x_{2}, \ldots, x_{n}\right)=\alpha_{0}+\sum_{i 1=1}^{n} \alpha_{i 1} \psi^{1}\left(x_{i 1}\right)+\sum_{i 1=1}^{n} \alpha_{i, i 1} \psi^{2}\left(x_{i 1}\right)+\sum_{1 \leq i 1<i 2 \leq n}^{n} \alpha_{i 1, i 2} \psi^{1}\left(x_{i 1}\right) \psi^{1}\left(x_{i 2}\right)+\left(\sum_{i 1=1}^{n} \alpha_{i 1, i 1, i 1} \psi^{3}\left(x_{i 1}\right)\right. \\
& \left.+\sum_{1 \leq i<i<\leq n}^{n} \alpha_{1, i, i, i} \psi^{2}\left(x_{i 1}\right) \psi^{1}\left(x_{i 2}\right)+\sum_{1 \leq i 1<i 2 \leq n}^{n} \alpha_{i 1, i 2, i 2} \psi^{1}\left(x_{\mathrm{i}}\right) \psi^{2}\left(x_{i 2}\right)+\sum_{1 \leq i<i 2<i 3 \leq n}^{n} \alpha_{i 1, i 2, i 3} \psi^{1}\left(x_{i 1}\right) \psi^{1}\left(x_{i 2}\right) \psi^{1}\left(x_{i 3}\right)\right)+\ldots
\end{aligned}
$$

Where the coefficients $\alpha$ are unknown, which are used to quantify the contribution of individual uncertain parameters or the interactions between/among uncertain parameters to the uncertainty of $T_{e} ; \psi$ is the optimal orthogonal basis for individual input parameters, whose superscript indicates the degree.

For computational purposes, the Eq. 2 should be truncated with a certain degree $d$ [18], as shown in Eq. 3 .

$$
\begin{aligned}
& T_{e}\left(x_{1}, x_{2}, \ldots, x_{n}\right) \approx \alpha_{0}+\sum_{i 1=1}^{n} \alpha_{i 1} \psi^{1}\left(x_{i 1}\right)+\sum_{i 1=1}^{n} \alpha_{i, i 1} \psi^{2}\left(x_{i 1}\right)+\sum_{1 \leq i<i 2 \leq n}^{n} \alpha_{i 1, i 2} \psi^{1}\left(x_{i 1}\right) \psi^{1}\left(x_{i 2}\right)+\cdots+ \\
& \left(\sum_{i 1=1}^{n} \alpha_{i 1 ; i, i} \psi^{d}\left(x_{i 1}\right)+\sum_{1 \leq i<i 2 \leq n} \alpha_{i \mathbb{R}} \psi^{d-1}\left(x_{i 1}\right) \psi^{1}\left(x_{i 2}\right)+\cdots\right)
\end{aligned}
$$

Where the number of unknown coefficients $a$ can be determined by $n$ and $d$, which is equal to $\frac{(n+d) !}{n ! d !}$.

The optimal orthogonal basis $\psi$ can be determined according to the Gram-Schmidt orthogonalization [10]. Based on the Gram-Schmidt orthogonalization, Oladyshkin [19] gave the relationship between optimal orthogonal basis and statistical moments of uncertain input parameters, whose distributions can be arbitrary with limited statistical moments. And the main steps to obtain the optimal orthogonal basis are summarized as follows: Firstly, for ease of analytical development, all uncertain parameters $x_{i}$ are transformed linearly to $x_{i}^{\prime}$ with 0 mean value and unit variance, as shown in Eq.(4).

$$
x_{i}^{\prime}=\frac{x_{i}-\mathrm{E}\left(x_{i}\right)}{\sqrt{\operatorname{Var}\left(x_{i}\right)}} \quad i=1,2, \cdots, n
$$

Where $\mathrm{E}\left(x_{i}\right)$ and $\operatorname{Var}\left(x_{i}\right)$ are the mean and variance of $x_{i}$. 
Secondly, the optimal orthogonal basis $\psi\left(x_{i}^{\prime}\right)$ can be obtained by solving the Eq. (14) in Ref. [19], which involves the statistical moments of $x_{i}^{\prime}$.

Finally, for more useful properties, the obtained $j$-th degree optimal orthogonal polynomial $\psi^{j}\left(x_{i}^{\prime}\right)$ is normalized as follows.

$$
\phi^{j}\left(x_{i}^{\prime}\right)=\frac{\psi^{j}\left(x_{i}^{\prime}\right)}{\int\left(\psi^{j}\left(x_{i}^{\prime}\right)\right)^{2} p\left(x_{i}^{\prime}\right) d x^{\prime}} \quad i=1,2, \cdots, n ; j=1,2, \cdots, d
$$

Where $\phi^{j}\left(x_{i}^{\prime}\right)$ is the $j$-th degree normalized orthogonal polynomial for $x_{i}^{\prime} ; p\left(x_{i}^{\prime}\right)$ is the probability density function for $x_{i}^{\prime}$, which is equal to the probability density function of $x_{i}$ due to the linear transformation between $x_{i}$ and $x_{i}^{\prime}$.

\section{Determination of Sample Points}

Unknown coefficients $\alpha$ can be solved by the regression method [18], which is associated with sample points of the input and output. For a certain uncertain input parameter, the optimal input sample points are the roots of the orthogonal polynomial of one degree higher than that used in the expansion [20]. For the $d$ th degree expansion with $n$ uncertain input parameters, the number of the optimal input sample points is $(d+1)^{n}$, which may be much larger than necessary. In order to avoid the unnecessary simulations and assure the accuracy, Isukapalli et al. [9] proposed two methods to select necessary input samples from the set of optimal input samples, i.e. selecting randomly and selecting based on probability distributions. For the former, the input sample size is recommended to be twice as many as the number of unknown coefficients. For the latter, the optimal input samples in high probability regions are given high priorities. In this paper, the latter is adopted to determine the necessary input sample points. Afterwards, the FDS+Evac model is performed on the necessary input sample points to generate the corresponding $T_{e}$ sample points. Once the input and output sample points are obtained, unknown coefficients $\alpha$ can be acquired by solving Eq. (3) using the singular value decomposition algorithm.

\section{Determination of the Appropriate Degree for the Surrogate Model of Evacuation Time}

When the optimal normalized orthogonal basis $\phi$ and unknown coefficients $\alpha$ are obtained, the $d$-th degree polynomial representation of $T_{e}$ can be determined. However, it is necessary to verify the degree for $T_{e}$ with the purpose of assuring the quality of the results.

In the range of a certain design parameter, the suitable degree for $T_{e}$ can be determined as follows: firstly, the design parameter is assumed to be distributed uniformly in its range. Afterwards, the $d$-th and $(d+1)$-th degree arbitrary polynomial chaos expansions are constructed, whose input parameters are uncertain parameters and the considered design parameter. And the design parameter can be regarded to be distributed uniformly in its design range here. Then, the cumulative distribution functions (CDFs) of $T_{e}$ can be numerically generated by combining LHS and the $d$-th and $(d+1)$-th degree arbitrary chaos expansions. If the difference between two CDFs is smaller than $5 \%$, the $(d+1)$-th degree is considered to be appropriate for the construction of the surrogate model of $T_{e}$ in the range of the design parameter. If the difference is significant, the $(d+2)$-th degree polynomial chaos expansion of $T_{e}$ should be reconstructed, and the above process should be repeated until two CDF curves are similar.

Here, the coefficient of variation of the root-mean-square deviation, CV (RMSD) is adopted to quantify the difference between two CDF curves of $T_{e}$. The larger the value of CV(RMSD) is, the more significant the difference between two CDF curves is, and CV(RMSD) can be expressed as follows. 


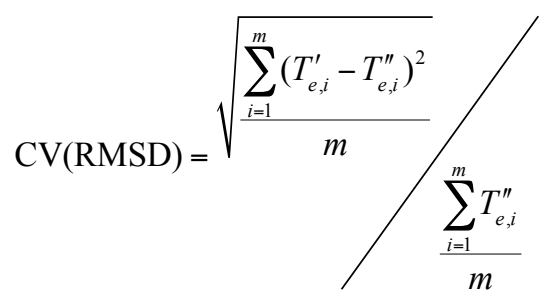

Where $m$ is the number of points constituting the CDF of $T_{e} ; T_{e, i}^{\prime}$ is the (i/m*100)-th percentile point for one CDF curve of $T_{e} ; T_{e, i}^{\prime \prime}$ is the (i/m*100)-th percentile point for another CDF curve of $T_{e}$ based on the arbitrary polynomial chaos expansion of one degree higher than that used to obtain $T_{e, i}^{\prime}$.

\section{Post-Processing}

Once the suitable degree for surrogate models of $T_{e}$ is determined in the range of a certain design parameter, the surrogate model of $T_{e}$ associated with uncertain input parameters can be constructed repeatedly at different values of the considered design parameter.

For a certain value of the design parameter, the surrogate model of $T_{e}$ with one suitable degree $d^{\prime}$ can be represented with the normalized orthogonal basis $\phi$ and coefficients $\alpha^{\prime}$, as shown in Eq. 7 .

$$
\begin{aligned}
& T_{e}\left(x_{1}^{\prime}, x_{2}^{\prime}, \ldots, x_{n}^{\prime}\right) \approx \alpha_{0}^{\prime}+\sum_{i 1=1}^{n} \alpha_{i 1}^{\prime} \phi^{1}\left(x_{i 1}^{\prime}\right)+\sum_{i 1=1}^{n} \alpha_{i 1, i 1}^{\prime} \phi^{2}\left(x_{i 1}^{\prime}\right)+\sum_{1 \leq i 1<i 2 \leq n} \alpha_{i 1, i 2}^{\prime} \phi^{1}\left(x_{i 1}^{\prime}\right) \phi^{1}\left(x_{i 2}^{\prime}\right)+\cdots+ \\
& \left(\sum_{i 1=1}^{n} \alpha_{i 1, i 1, \ldots, i 1}^{\prime} \phi^{d^{\prime}}\left(x_{i 1}^{\prime}\right)+\sum_{1 \leq i<i<\leq n} \alpha_{i 1, i 2, \cdots, i 2}^{\prime} \phi^{\left(d^{\prime}-1\right)}\left(x_{i 1}^{\prime}\right) \phi^{1}\left(x_{i 2}^{\prime}\right)+\cdots\right)
\end{aligned}
$$

Furthermore, due to the orthogonal and normalized properties of $\phi$, the mean and variance of $T_{e}$, $\mathrm{E}\left(T_{e}\right)$ and $\operatorname{Var}\left(T_{e}\right)$ can be analytically calculated as follows.

$$
\begin{aligned}
& \mathrm{E}\left(T_{e}\right)=\alpha_{0}^{\prime} \\
& \operatorname{Var}\left(T_{e}\right)=\sum_{i 1=1}^{n}\left(\alpha_{i 1}^{\prime}\right)^{2}+\sum_{i 1=1}^{n}\left(\alpha_{i 1, i 1}^{\prime}\right)^{2}+\sum_{1 \leq i 1<i 2 \leq n}\left(\alpha_{i 1, i 2}^{\prime}\right)^{2}+\cdots+\left(\sum_{i 1=1}^{n}(\underbrace{\alpha_{i 1, i 1, \ldots, i 1}^{\prime}}_{d^{\prime}})^{2}+\sum_{1 \leq i 1<i 2 \leq n}(\underbrace{\left.\alpha_{d}^{\prime}-1,1\right)}_{\underbrace{\prime}_{i 1, i 1, \cdots, i 1, i 2}})^{2}+\cdots\right)
\end{aligned}
$$

Based on the definition of Sobol sensitivity indices [21], the main and total sensitivity indices of $T_{e}$ for uncertain parameter $x_{i}, S_{x_{i}}$ and $S_{x_{i}}^{T}$ can be expressed as follows.

$$
\begin{aligned}
S_{x_{i}} & =\frac{\operatorname{Var}\left(\mathrm{E}\left(T_{e} \mid x_{i}\right)\right)}{\operatorname{Var}\left(T_{e}\right)} \\
S_{x_{i}}^{T} & =\frac{\operatorname{Var}\left(T_{e}\right)-\operatorname{Var}\left(\mathrm{E}\left(T_{e} \mid \mathbf{x}_{-\mathrm{i}}\right)\right)}{\operatorname{Var}\left(T_{e}\right)}=\frac{\mathrm{E}\left(\operatorname{Var}\left(T_{e} \mid \mathbf{x}_{-\mathrm{i}}\right)\right)}{\operatorname{Var}\left(T_{e}\right)}
\end{aligned}
$$

Where $\mathrm{E}\left(T_{e} \mid x_{i}\right)$ is the mean value (expected value) of $T_{e}$ given the values of $x_{i}$, whose variance $\operatorname{Var}\left(\mathrm{E}\left(T_{e} \mid x_{i}\right)\right)$ is equal to the variance of $\mathrm{E}\left(T_{e} \mid x_{i}^{\prime}\right)$ due to the linear transformation of $x_{i} ; \mathrm{E}\left(T_{e} \mid \mathbf{x}_{-\mathrm{i}}\right)$ is the mean value (expected value) of $T_{e}$ given the values of non- $x_{i} ; \operatorname{Var}\left(T_{e} \mid \mathbf{x}_{-\mathrm{i}}\right)$ is the variance of $T_{e}$ given the 
values of non- $x_{i}$, whose mean value (expected value) $\mathrm{E}\left(\operatorname{Var}\left(T_{e} \mid \mathbf{x}_{-\mathrm{i}}\right)\right)$ is equal to the mean value of $\operatorname{Var}\left(T_{e} \mid \mathbf{x}_{-\mathrm{i}}^{\prime}\right)$ due to the linear transformation of $x_{i}$.

Due to the orthogonality and normalization of $\phi$, the variance of $\mathrm{E}\left(T_{e} \mid x_{i}^{\prime}\right)$ and the mean of $\operatorname{Var}\left(T_{e} \mid \mathbf{x}_{-\mathrm{i}}^{\prime}\right)$, $\operatorname{Var}\left(\mathrm{E}\left(T_{e} \mid x_{i}^{\prime}\right)\right)$ and $\mathrm{E}\left(\operatorname{Var}\left(T_{e} \mid \mathbf{x}_{-\mathrm{i}}^{\prime}\right)\right)$ can be analytically computed, as shown in Eq. (10) and Eq. (11).

$$
\begin{aligned}
& \operatorname{Var}\left(\mathrm{E}\left(T_{e} \mid x_{i}^{\prime}\right)\right)=\sum_{i 1=i}\left(a_{i 1}^{\prime}\right)^{2}+\sum_{i 1=i}\left(a_{i 1, i 1}^{\prime}\right)^{2}+\ldots+\sum_{i 1=i}\left(a_{d^{\prime}}^{a_{i 1, i 1, \cdots, i 1}^{\prime}}\right)^{2} \\
& \mathrm{E}\left(\operatorname{Var}\left(T_{e} \mid \mathbf{x}_{-\mathrm{i}}^{\prime}\right)\right)=\sum_{i 1=i}\left(a_{i 1}^{\prime}\right)^{2}+\sum_{i 1=i}\left(a_{i 1, i 1}^{\prime}\right)^{2}+\sum_{1 \leq i<<i=i \leq n}\left(a_{i 1, i 2}^{\prime}\right)^{2}+\sum_{1 \leq i 1=i<i 2 \leq n}\left(a_{i 1, i 2}^{\prime}\right)^{2}+\cdots \\
& +\left(\sum_{i 1=i}(\underbrace{a_{i 1, i 1, \ldots, i 1}^{\prime}}_{d^{\prime}})^{2}+\sum_{1 \leq i<<i 2=i \leq n}(\underbrace{\left.a^{\prime}-1\right)}_{d^{i 1, \ldots, i 1, i 2}})^{2}+\sum_{1 \leq i \leq i=i<i 2 \leq n}\left(a_{a^{i 1, \ldots, i 1, i 2}}^{\left.a^{\prime}-1\right)}\right)^{2}+\cdots\right)
\end{aligned}
$$

Moreover, the CDFs of $T_{e}$ can be numerically generated by combining surrogate models of $T_{e}$ and LHS. For different values of the design parameter, $T_{e}$ at any level of uncertainty of interest can be obtained from the CDF of $T_{e}$. Based on the description above, the effect of the design parameter on $T_{e}$ under arbitrary uncertainty can be examined.

The application of the arbitrary polynomial chaos-based method is demonstrated in the following case study, which is hypothetical due to the difficulty in obtaining the available experimental data of evacuation and scenarios.

\section{CASE STUDY AND ANALYSIS}

\section{Case Description}

The case considered is a single-room fire compartment of civil buildings, which is enclosed by fireresistant construction to prevent the spread of fire to adjacent rooms. In order to make the considered fire compartment representative, the fire compartment is set to be $2500 \mathrm{~m}^{2}$, which is the maximum area of one fire compartment of civil buildings regulated by the Chinese code GB50016-2012 [22]. Meanwhile, the minimum number of exits is 2 for this considered fire compartment prescribed by GB50016-2012. Given a worse case, 2 exits, denoted by Exit A and Exit B with the same size, are located at the centre of the walls. Additionally, in order to decrease the computational cost of evacuation simulations, obstacles are not taken into consideration in this fire compartment. Meanwhile, given emergency evacuation, occupants will evacuate immediately while notified by fire alarm systems. Thus, the pre-movement times for the crowd are assumed to $0 \mathrm{~s}$ here. Moreover, there is a possibility that fire may occur around one exit. Here, it is assumed that Exit B is not available while fire occurs. The plan of this considered fire compartment is shown in Fig.2.

Due to the randomness of fire occurrence, it is difficult to accurately acquire the number and characteristics of evacuees. Thus, two uncertain parameters are considered here: occupant density and child-occupant load ratio. Compared with low density crowd evacuation, there is a higher risk in high density crowd evacuation. Here, the mean value of occupant density is assumed to be 1.0 person $/ \mathrm{m}^{2}$. Based on the occupant type of "School Children" defined by the SIMULEX evacuation model [23], the mean value of the child-occupant load ratio is assumed to be 0.7 here. Moreover, occupant density and the child-occupant load ratio are assumed to follow normal distributions, which are truncated due to the bounded property of these parameters. Meanwhile, the standard deviations of normal distributions for occupant density and the child- 
occupant load ratio are assumed to be $20 \%$ and $10 \%$ of their respective mean values. Table 1 gives the distributions of occupant density and child-occupant load ratio used in this case.

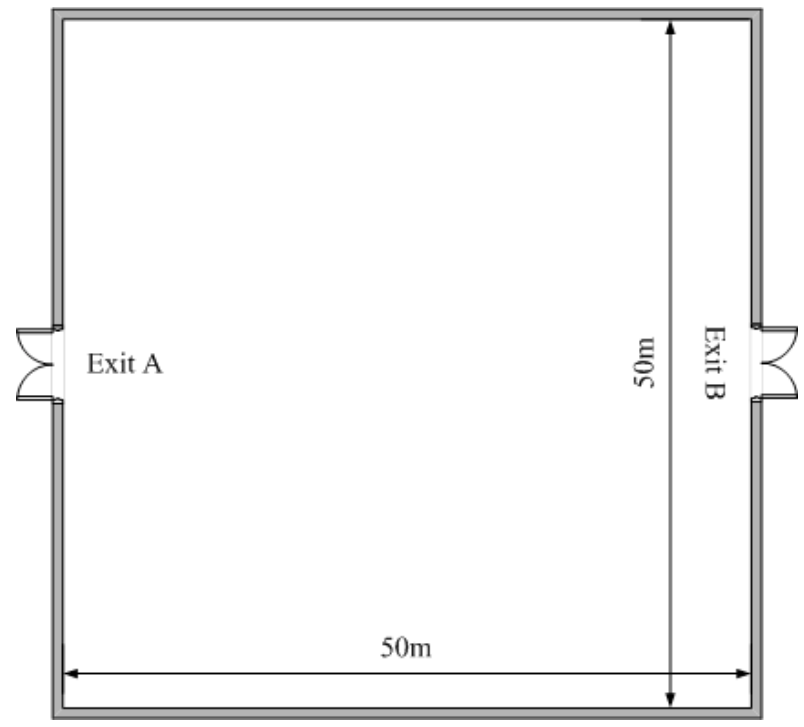

Fig. 2. The plan of the fire compartment considered.

Table 1. Distributions of uncertain parameters considered.

\begin{tabular}{|c|c|}
\hline Uncertain parameters & Probability distribution functions \\
\hline $\begin{array}{c}\text { Occupant density, } \\
x_{1} /\left(\text { persons } / \mathrm{m}^{2}\right)\end{array}$ & Truncated normal distribution: \\
\hline & $f\left(x_{1}\right)=\frac{1}{\sqrt{2 \pi} \sigma} \exp \left[-\frac{\left(x_{1}-u\right)^{2}}{2 \sigma^{2}}\right]$ with $u=1, \sigma=0.2,0.8 \leq x_{1} \leq 1.2$ \\
Child-Occupant load ratio, & Truncated normal distribution: \\
$x_{2}$ & $f\left(x_{2}\right)=\frac{1}{\sqrt{2 \pi} \sigma} \exp \left[-\frac{\left(x_{2}-u\right)^{2}}{2 \sigma^{2}}\right]$ \\
& with $u=0.7, \sigma=0.07,0.56 \leq x_{2} \leq 0.84$ \\
\hline
\end{tabular}

In performance-based fire protection design of buildings, exit width is generally paid great attention to by fire engineers or designers. When exit width is too small, people will not evacuate successfully. When exit width is too large, the available area of use will be reduced significantly. Here, the maximum and minimum exit widths are determined based on $0.5 \mathrm{~m}$ per 100 people [24] and $0.1 \mathrm{~m}$ per 100 persons, respectively. According to the analysis above, the design range of exit width is determined, as shown in Table 2.

Table 2. Design parameter in the case.

\begin{tabular}{|c|c|}
\hline Design parameter & Range \\
\hline Width for Exit A $/ \mathrm{m}$ & $2.5^{\mathrm{a}}-12.5^{\mathrm{b}}$ \\
\hline
\end{tabular}

${ }^{\mathrm{a}} 2.5$ is calculated by the area of this fire compartment $2500 \mathrm{~m}^{2}$ multiplied by occupant density 1.0 person $/ \mathrm{m}^{2}$ multiplied by $0.001 \mathrm{~m} /$ person.

${ }^{b} 12.5$ is calculated by the area of this fire compartment $2500 \mathrm{~m}^{2}$ multiplied by occupant density 1.0 person $/ \mathrm{m}^{2}$ multiplied by $0.005 \mathrm{~m} /$ person that is chosen according to Ref. [24].

According to the proposed method, we should firstly determine the suitable degree for surrogate models of $T_{e}$ associated with occupant density and child-occupant load ratio in the design range of exit width. Afterwards, surrogate models of $T_{e}$ can be constructed repeatedly for different values of exit width. Finally, 
the analytical and numerical methods are used as post-processing to examine the effect of exit width on the uncertainty of $T_{e}$ caused by occupant density and child-occupant load ratio.

\section{Results and Discussion}

In order to determine the suitable degree for surrogate models of $T_{e}$ in 2.5-12.5 m exit width, the $2^{\text {nd }}, 3^{\text {rd }}$ and $4^{\text {th }}$ degree arbitrary polynomial chaos expansions of $T_{e}$ are constructed based on 16,41 and 86 samples respectively, whose input parameters are occupant density, child-occupant load ratio and exit width. Then, the LHS is applied to occupant density, child-occupant load ratio, and exit width to obtain input samples. Afterwards the CDFs of $T_{e}$ can be obtained by performing the $2^{\text {nd }}, 3^{\text {rd }}$ and $4^{\text {th }}$ degree expansions on these input sample points, as shown in Fig.3. For the $2^{\text {nd }}$ and $3^{\text {rd }}$ degree expansions, the value of CV(RMSD) between two CDF curves of $T_{e}$ is $8.12 \%$. However, for the $3^{\text {rd }}$ and $4^{\text {th }}$ degree expansions, it is $2.78 \%$ (smaller than $5 \%$ ), which suggests that the $4^{\text {th }}$ degree is suitable for the construction of surrogate models of $T_{e}$ in the design range of exit width. Furthermore, in order to verify the proposed method, the MCS can be applied to the FDS+Evac model. The sample size of the MCS that can be estimated by statistical tolerance limits [25]. For the statistical tolerance limits $(99.5 \%$, 99.5\%), which indicates that with the confidence level of $99.5 \%, 99.5 \%$ of the samples are in the tolerance limits, the corresponding sample size is estimated around 1000. Thus, the MCS of the FDS+Evac model with 1000 sample size is performed in this case, and the corresponding CDF of $T_{e}$ is also shown in Fig. 3. And the value of CV(RMSD) between two CDF curves, which are based on the $4^{\text {th }}$ degree expansion of $T_{e}$ with 86 samples and the MCS with 1000 samples, is $1.86 \%$ (smaller than $5 \%$ ), which suggests that the $4^{\text {th }}$ arbitrary polynomial chaos expansion of $T_{e}$ is around a $99.5 \%$ confidence level. Thus in the range of 2.5-12.5 m exit width, the appropriate degree for surrogate models of $T_{e}$ is 4 in this case study.

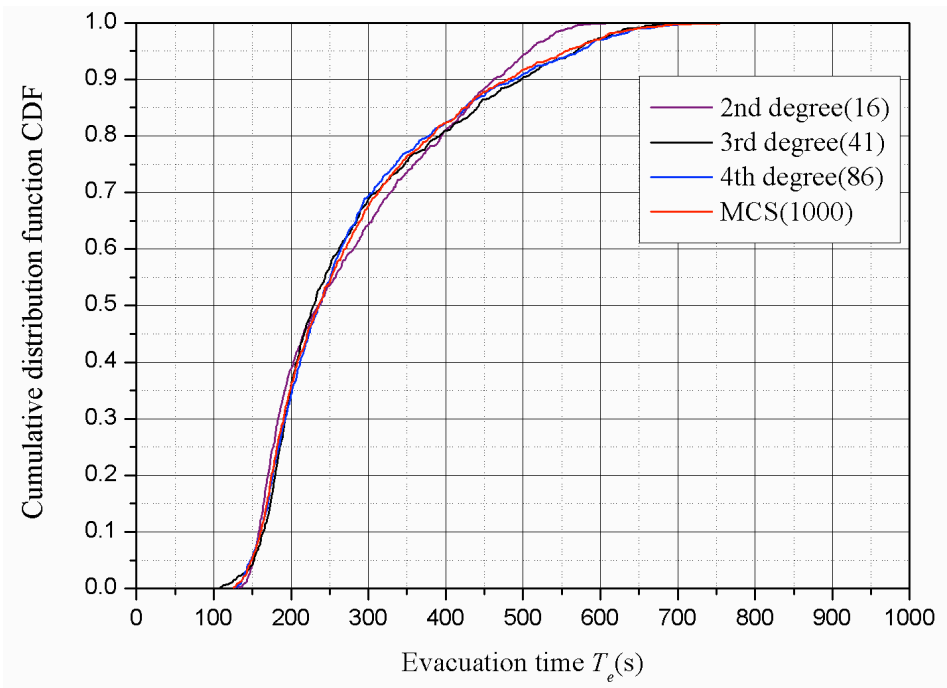

Fig. 3. Cumulative distribution functions (CDFs) of evacuation time $T_{e}$ based on the $2^{\text {nd }}, 3^{\text {rd }}$ and $4^{\text {th }}$ degree arbitrary polynomial chaos expansions and Monte Carlo simulation (MCS).

For a certain fixed value of exit width, 18 samples are required to construct one arbitrary polynomial chaos expansion of the $4^{\text {th }}$ degree for $T_{e}$, whose input parameters are occupant density and child-occupant load ratio in this case. To investigate the effect of exit width on evacuation time under uncertainty, the $4^{\text {th }}$ degree expansions of $T_{e}$ need to be constructed repeatedly for different values of exit width. Afterwards, the corresponding mean value and standard deviation of $T_{e}$ as well as Sobol total sensitivity indices can be calculated by analytical methods according to Eq.(8) and Eq.(9), as shown in Fig. 4.

From Fig. 4 (a) and (b), it can be seen that the mean and standard deviation of $T_{e}$ decrease with the increase of exit width. For example, when exit width is $2.5 \mathrm{~m}$, the mean value and standard deviation of $T_{e}$ are 664.0 $\mathrm{s}$ and $71.0 \mathrm{~s}$. However, when exit width is $12.5 \mathrm{~m}$, the mean and standard deviation of $T_{e}$ are $152.0 \mathrm{~s}$ and $15.2 \mathrm{~s}$, which are much smaller than those for $2.5 \mathrm{~m}$ exit width. The explanation about the results is that while increasing exit width, the interaction among evacuees around exits will decrease, and congestion and 
queuing phenomena around exits will be eased, which also suggests that the effect of occupant density and child-occupant load ratio on $T_{e}$ will decrease with the increase of exit width. Thus while increasing the exit width, $T_{e}$ for a certain deterministic evacuation scenario will decrease and the difference in $T_{e}$ for different scenarios will not be significant due to the decrease in the effects of occupant density and child-occupant load ratio on $T_{e}$. From the analysis above, it can be seen that the mean value and standard deviation of $T_{e}$ for uncertain evacuation scenarios will decrease with the increase of exit width. Moreover, when exit width is large, there are still strong interactions for high density crowd who are far away from exits during the movement process, which suggests that there are still some differences in $T_{e}$ for different scenarios of high density crowd evacuation. From the analysis above, it can be deduced that when exit width is large enough, the mean value and standard deviation of $T_{e}$ for high density crowd will almost be unchanged with exit width. Furthermore, the Sobol total sensitivity indices are adopted here to quantify the contributions of occupant density and child-occupant load ratio to the uncertainty of $T_{e}$, as shown in Fig. 4 (c) and (d). From Fig. 4 (c) and (d), it can be seen that the contributions of occupant density and child-occupant load ratio to $T_{e}$ are little affected by exit width. Moreover, compared with the child-occupant load ratio, the contribution of occupant density to the uncertainty of $T_{e}$ is much larger. Thus, for the high density crowd evacuation, the child-occupant load ratio can be taken as the base value, whose uncertainty can be ignored.
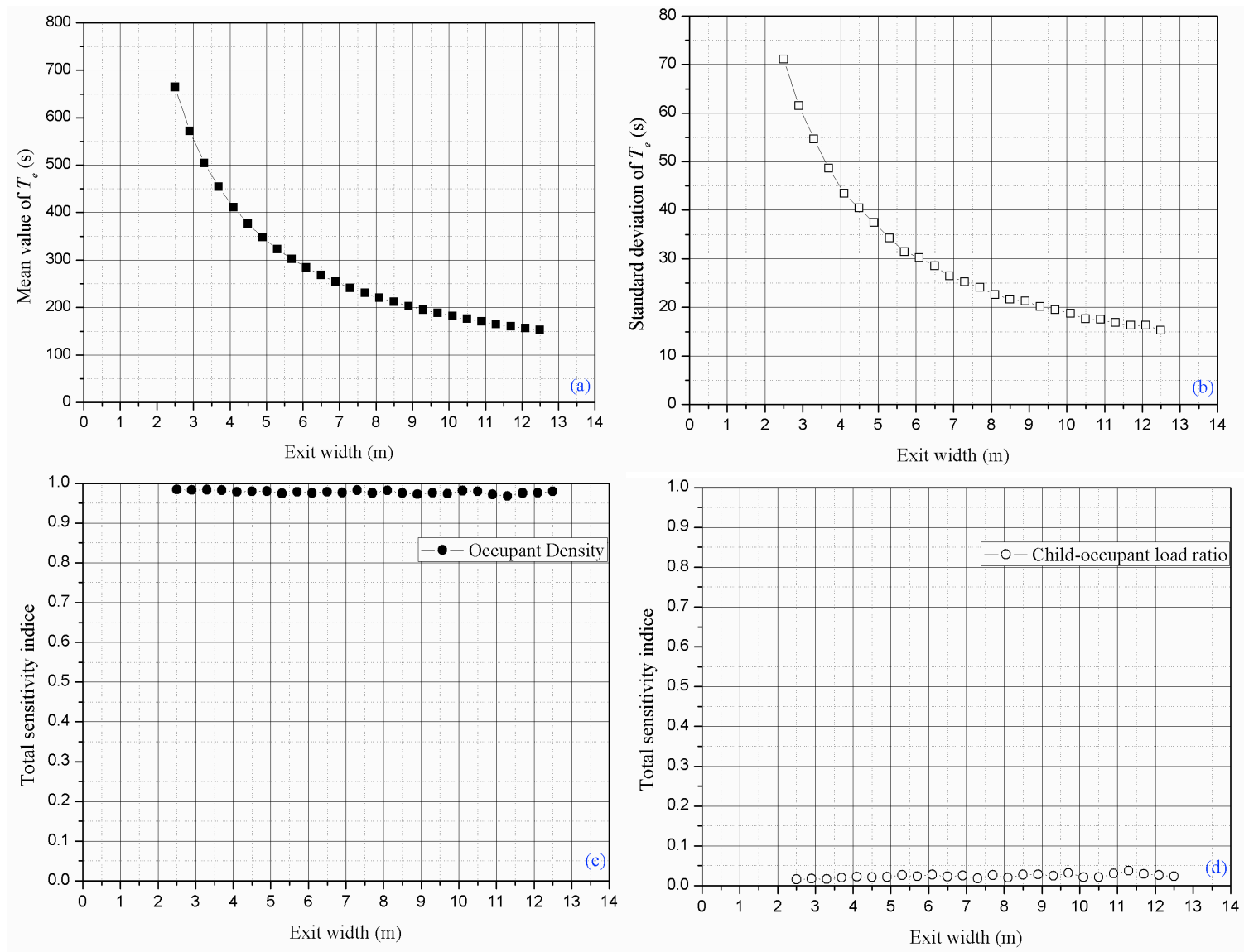

Fig. 4. Mean value (a), and standard deviation (b) of evacuation time $T_{e}$ as well as Sobol total sensitivity indices for occupant density (c) and child-occupant load ratio (d) versus exit width.

Exit width and the uncertainty of evacuation time are key considerations in performance-based fire safety design. In order to obtain an optimal exit width under uncertainty, the effect of exit width on the distribution of $T_{e}$ need to be investigated. Considering evacuation times of interest, the base case, $80^{\text {th }}$ percentile, $90^{\text {th }}$ percentile and $99.9^{\text {th }}$ percentile evacuation times are given in Fig.5. Here, the base case evacuation time is calculated from the $4^{\text {th }}$ degree polynomial chaos expansions of $T_{e}$, whose uncertain parameters are taken as deterministic average values. The limitations of the RSET (required safety egress 
time)/ASET (available safety egress time) approach are widely known [26]. ASET may vary from 60-90 s in ultra-fast fire to 6-9 $\mathrm{min}$ in moderately fast growing fire [26]. The proper ASET value shall be the object of study considering the characteristics of each building, as a function of the inflammable material amount, type and distribution. In this case study, the exit width prescribed by the Chinese code GB50016-2012 (around $16.2 \mathrm{~m}$ wide for shopping malls) leads to a RSET around $120 \mathrm{~s}$, corresponding to an ultra-fast growth rate fire. This exit may be oversized if there is no enough combustible material in this building. In addition, for a certain acceptable ASET, the optimal exit width at a certain reliability level can be determined from Fig. 5. For example, when the acceptable ASET is $300 \mathrm{~s}$, the optimal exit width at the reliability level of $99.9 \%$ should be selected around $7.0 \mathrm{~m}$ based on Fig. 5. Furthermore, for a certain exit width, $T_{e}$ at a certain reliability level can also be obtained from Fig. 5. For example, when exit width is around $10 \mathrm{~m}, T_{e}$ at the reliability probability of $99.9 \%$ should be around $218 \mathrm{~s}$ in this case. If the corresponding ASET is smaller than $218 \mathrm{~s}$, some measures should be taken, such as improving the detection and alarm system, installing the fire extinction and smoke control systems.

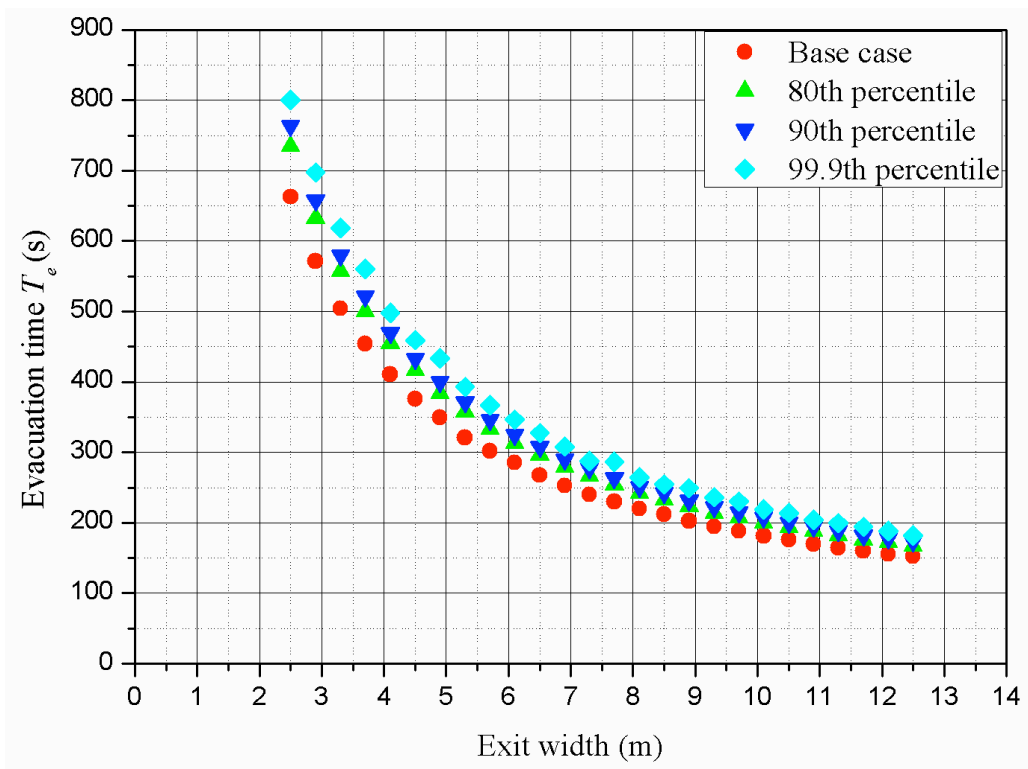

Fig. 5. Evacuation time $T_{e}$ under uncertainty versus exit width.

Due to the significant computational cost of the MCS for complex evacuation models, the safety factor method is generally adopted to deal with the uncertainty of evacuation time in performance-based fire protection design of buildings. However, the uncertainty of evacuation time is affected by various factors such as occupancy type, exit width, and the characteristics of evacuees, which makes the safety factor random. Thus, the effect of exit width on uncertainty factor [27], which is defined as the ratio of evacuation time under uncertainty to the base case evacuation time, is investigated, as shown in Fig. 6. For a specified exit width, the optimal safety factor at a certain reliability level can be determined from Fig. 6. For example, when exit width is $8.5 \mathrm{~m}$, the optimal safety factor at the reliability level of $99.9 \%$ is 1.20 . In addition, for a specified exit width, the reliability probability of a certain safety factor can also be determined from Fig. 6 . E.g. when exit width is $4.5 \mathrm{~m}$, the reliability probability for the safety factor of 1.15 is $90 \%$. From Fig.6, it can also be seen that in the design range of $0.1 \mathrm{~m}$ and $0.5 \mathrm{~m}$ per 100 persons, exit width has little effect on the uncertainty factor at a certain reliability level and the reliability level of a certain uncertainty factor, which suggests that the safety factor at a certain reliability level and the reliability level of a certain safety factor are almost independent of exit width. 


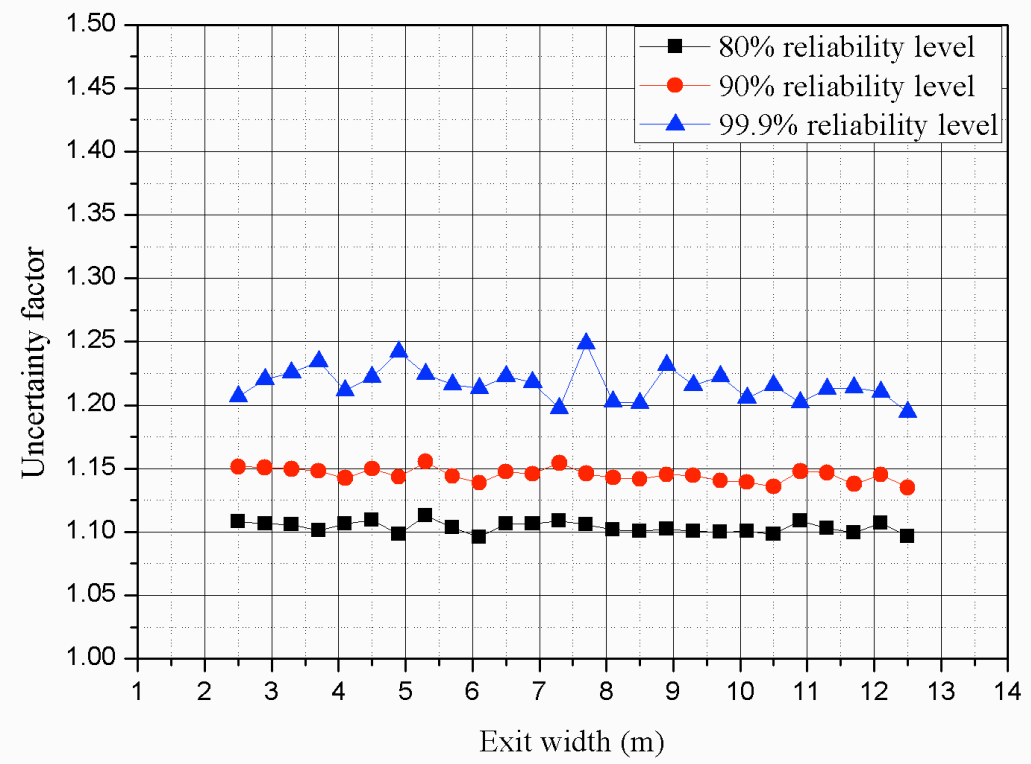

Fig. 6. Uncertainty factor versus exit width.

\section{CONCLUSIONS}

In order to conduct a cost-effective performance-based fire protection design of buildings with the reduced computational cost, an arbitrary polynomial chaos-based method is presented to examine the effects of design parameters on evacuation time under arbitrary uncertainty in this work. Based on a hypothetical case study of a single-room fire compartment with two exits, some conclusions can be summarized as follows.

The proposed method is demonstrated by the issue of the effect of exit width on the uncertainty of evacuation time resulting from the uncertainty of occupant density and child-occupant load ratio. Results reveal that the arbitrary polynomial chaos-based method can be used to investigate the impacts of design parameters on evacuation time under arbitrary uncertainty, whose computational cost is reduced significantly compared with the conventional MCS. Besides, the mean and standard deviation of evacuation time as well as Sobol sensitivity indices can be analytically calculated on the basis of the arbitrary polynomial chaos expansion coefficients, which does not need extra evacuation simulations.

From this case, it can be seen that the uncertainty of evacuation time is significantly affected by exit width designed between $0.1 \mathrm{~m}$ and $0.5 \mathrm{~m}$ per 100 persons, which is more significant with smaller exit width. Moreover, in this considered design range, the contributions of occupant density and child-occupant load ratio to the uncertainty of evacuation time are not severely affected by exit width. Meanwhile, the safety factor at a certain reliability level and the reliability level of a certain safety factor are almost independent of exit width. This method can also be used to optimize other design parameters in performance-based design of buildings, such as exit position.

However, there are still some limitations in this proposed method. First of all, we construct surrogate models of evacuation time based on the simulations of complex evacuation models, which makes the accuracy of the proposed method depend to a considerable degree on the selected evacuation model. Thus, it should be prudent to choose the evacuation model to make constructed surrogate models of evacuation time accurate enough.

Secondly, this proposed method is applied to a hypothetical case, which has not been validated due to the difficulty in obtaining the available evacuation data. In the future, we will focus on designing and carrying out the realistic evacuation experiments to obtain the valuable evacuation data.

Thirdly, for multiple input parameters, it is computationally expensive to adopt the arbitrary polynomial chaos-based method to investigate the effect of the design parameter on evacuation time under uncertainty due to the significant increase of the number of necessary evacuation simulations with the number of input parameters. 
Finally, the proposed method only can be used to deal with input parameters that are independent or linearly correlated. And the uncertainty of the output resulting from nonlinear uncertain input parameters need to be addressed in our future work.

\section{ACKNOWLEDGEMENT}

This work is sponsored by the Research Fund for the Doctoral Program of Higher Education of China (Grant No.20123402110048). And the numerical calculations in this work have been performed at the Supercomputing Center of University of Science and Technology of China. We would like to express our appreciation to its help and support. The first author also thanks Bruno Lee for his help with discussion.

\section{REFERENCES}

[1] Gwynne, S., Galea, E.R., Owen, M., and Lawrence, P.J., (1999) A Review of the Methodologies Used in the Computer Simulation of Evacuation from the Built Environment. Building and Environment 34: 741-749, http://dx.doi.org/10.1016/S0360-1323(98)00057-2

[2] Hadjisophocleous, G.V., Benichou, N., and Tamim, A.S., (1998) Literature Review of Performance-Based Fire Codes and Design Environment. Journal of Fire Protection Engineering 9(1): 12-40, http://dx.doi.org/10.1177/104239159800900102

[3] Meacham, B., Lord, J., Moore, A., Proulx, G., Fahy, R., and Notarianni, K., "Investigation of Uncertainty in Egress Models and Data," Proceedings of the 3rd International Symposium on Human Behaviour in Fire, 2004. pp. 419-428.

[4] Fang, Z.M., Song, W.G., Zhang, J., and Wu, H., (2012) A Multi-Grid Model for Evacuation Coupling with the Effects of Fire Products. Fire Technology 48(1): 1-14, http://dx.doi.org/10.1007/s10694-010-0173-x

[5] Zhao, D.L., Li, J., Zhu, Y., and Zou, L., (2008) The Application of a Two-Dimensional Cellular Automata Random Model to the Performance-Dased Design of Building Exit. Building and Environment 43(4): 518-522, http://dx.doi.org/10.1016/j.buildenv.2007.01.011

[6] Tavares, R.M. and Galea, E.R., (2009) Evacuation Modelling Analysis within the Operational Research Context: A Combined Approach for Improving Enclosure Designs. Building and Environment 44(5): 1005-1016, http://dx.doi.org/10.1016/j.buildenv.2008.07.019

[7] Tavares, R.M., (2010) Design for Horizontal Escape in Buildings: The Use of the Relative Distance between Exits as an Alternative Approach to the Maximum Travel Distance. Safety Science 48(10): 1242-1247, http://dx.doi.org/10.1016/j.ssci.2010.03.009

[8] Albrecht, C. and Hosser, D., "A Response Surface Methodology for Probabilistic Life Safety Analysis Using Advanced Fire Engineering Tools," Fire Safety Science--Proceedings of the Tenth Internatioinal Symposium, 2011. pp. 1059-1072.

[9] Isukapalli, S.S., Roy, A., and Georgopoulos, P.G., (1998) Stochastic Response Surface Methods (SRSMs) for Uncertainty Propagation: Application to Environmental and Biological Systems. Risk Analysis 18(3): 351-363, http://dx.doi.org/10.1111/j.1539-6924.1998.tb01301.x

[10] Witteveen, J.A.S. and Bijl, H., "Modeling Arbitrary Uncertainties Using Gram-Schmidt Polynomial Chaos," 44th AIAA Aerospace Sciences Meeting and Exhibit, 2006.

[11] Kuligowski, E.D. and Peacock, R.D., "A Review of Building Evacuation Models," National Institute of Standards and Technology Technical Note 1471, 2005.

[12] Schadschneider, A., Klingsch, W., Klüpfel, H., Kretz, T., Rogsch, C., and Seyfried, A., "Evacuation Dynamics: Empirical Results, Modeling and Applications," Extreme Environmental Events, R.A. Meyers, (ed.), Springer: New York, 2011 p. 517-550.

[13] Zheng, X., Zhong, T., and Liu, M., (2009) Modeling Crowd Evacuation of a Building Based on Seven Methodological Approaches. Building and Environment 44(3): 437-445, http://dx.doi.org/10.1016/j.buildenv.2008.04.002

[14] Ronchi, E., Colonna, P., and Berloco, N., (2013) Reviewing Italian Fire Safety Codes for the Analysis of Road Tunnel Evacuations: Advantages and Limitations of Using Evacuation Models. Safety Science 52(0): 28-36, http://dx.doi.org/10.1016/j.ssci.2012.03.015

[15] Korhonen, T. and Hostikka, S., "Fire Dynamics Simulator with Evacuation: FDS+ Evac, Technical Reference and User's Guide," VTT Technical Research Centre of Finland, 2010. 
[16] Wagner, S., "Global Sensitivity Analysis of Predictor Models in Software Engineering," PROMISE '07 Proceedings of the Third International Workshop on Predictor Models in Software Engineering, IEEE, 2007.

[17] Wiener, N., (1938) The Homogeneous Chaos. American Journal of Mathematics 60(4): 897-936, http://dx.doi.org/doi:10.2307/2371268

[18] Sudret, B., (2008) Global Sensitivity Analysis Using Polynomial Chaos Expansions. Reliability Engineering and System Safety 93(7): 964-979, http://dx.doi.org/10.1016/j.ress.2007.04.002

[19] Oladyshkin, S. and Nowak, W., (2012) Data-Driven Uncertainty Quantification Using the Arbitrary Polynomial Chaos Expansion. Reliability Engineering and System Safety 106(0): 179190, http://dx.doi.org/10.1016/j.ress.2012.05.002

[20] Fedorov, V.V., Theory of Optimal Experiments, Academic Press, New York, 1972.

[21] Homma, T. and Saltelli, A., (1996) Importance Measures in Global Sensitivity Analysis of Nonlinear Models. Reliability Engineering and System Safety 52(1): 1-17, http://dx.doi.org/10.1016/0951-8320(96)00002-6

[22] GB50016, "Code of Design on Building Fire Protection and Prevention," The Ministry of Public Security of the People's Republic of China, Beijing, China, 2012.

[23] IES, "Simulex User Guide," Integrated Environmental Solutions Limited, 2007.

[24] NFPA 101, " Life Safety Code," National Fire Protection Association,Quincy, MA, USA, 2010.

[25] Matala, A., "Sample Size Requirement for Monte Carlo-simulations using Latin Hypercube Sampling," Helsinki University of Technology, Department of Engineering Physics and Mathematics, Systems Analysis Laboratory, 2008.

[26] Babrauskas, V., Fleming, J.M., and Don Russell, B., (2010) RSET/ASET, a flawed concept for fire safety assessment. Fire and Materials 34(7): 341-355, http://dx.doi.org /10.1002/fam.1025

[27] Xie, Q., Lu, S., Kong, D., and Wang, J., (2013) Treatment of Evacuation Time Uncertainty Using Polynomial Chaos Expansion. Journal of Fire Protection Engineering 23(1): 31-49, http://dx.doi.org/10.1177/1042391512470578 